\title{
Preoperative Chemoradiation in Carcinoma Esophagus: Experience from a Tertiary Cancer Center in India
}

\begin{abstract}
Background: Esophageal cancer is the fourth most common cause of cancer-related deaths in India. In 2012, the CROSS trial evaluated the benefit of induction therapy using weekly carboplatin-paclitaxel with 41.4 Gy radiation versus surgery alone. Pathological complete response (pCR) rates were $28 \%$. Overall survival was improved in the combined therapy arm. However, there is limited data regarding the outcomes of preoperative chemoradiation in Indian scenario. This study was conducted to determine the response to preoperative chemoradiation at a tertiary cancer center in India. Aims: Primary objective was to evaluate the rates of pCR. Secondary objective was to determine recurrence rate and patterns of recurrence. Materials and Methods: We retrospectively reviewed patients with locally advanced esophageal cancer and gastroesophageal junction treated from September 2013 to July 2015. Patients who received preoperative radiotherapy 41.4 Gy with chemotherapy (weekly paclitaxel-carboplatin or cisplatin-5 FU or cisplatin-capecitabine) were included in this study. Chi-square test and Fischer's exact test were used for analysis. Results: Fifty patients were included in the analysis $(76 \%$ - squamous cell carcinoma [SCC] and $24 \%$ - adenocarcinomas [ACC]). About $60 \%$ patients received weekly paclitaxel-carboplatin (CROSS protocol), 32\% cisplatin-5 FU, and 8\% cisplatin-capecitabine. pCR rate was $39.6 \%$. pCR rate was higher in SCC versus ACC (44.7\% vs. $20 \%$ ) and in paclitaxel-carboplatin than cisplatin-5FU or cisplatin-capecitabine ( $48.3 \%$ vs. $25 \%$ vs. $33.3 \%)$. At a median follow-up of 12 months, 38 patients were alive, 8 died, and 4 lost to follow-up. Of the 10 patients that recurred, 8 were distant recurrences. Conclusion: Our study results show favorable pCR rate after preoperative CRT with significant higher rate in SCC and patients receiving CROSS regimen. Majority of the recurrences were distant recurrences.
\end{abstract}

Keywords: Carcinoma Esophagus, pathological complete response, preoperative chemoradiation

\section{Introduction}

Esophageal cancer is the fourth most common cause of cancer-related deaths in India. Squamous cell carcinoma is the most common histology $(80 \%)$ although there has been a recent relative increase in the incidence of adenocarcinoma. ${ }^{[1]}$

In 2012, a multi-institutional Phase III study (CROSS trial) evaluated the benefit of induction therapy using weekly carboplatin and paclitaxel with 41.4 Gy radiation versus surgery alone. ${ }^{[2]}$ The pathological complete response (pCR) rate was $28 \%$, and overall survival was much improved in the combined therapy arm. However, there is limited data regarding the outcomes of preoperative chemoradiation in Indian scenario. Esophageal cancer in India has a unique etiological basis (alternative forms

This is an open access journal, and articles are distributed under the terms of the Creative Commons Attribution-Non Commercial-ShareAlike 4.0 License, which allows others to remix, tweak, and build upon the work non-commercially, as long as appropriate credit is given and the new creations are licensed under the identical terms.

For reprints contact: reprints@medknow.com of tobacco and tea drinking). ${ }^{[1]}$ Most patients present in advanced stage and poor general health at diagnosis. This retrospective study was conducted to determine the response to preoperative chemoradiation at a tertiary care center in India.

\section{Aims}

Primary objective was to evaluate the rates of $\mathrm{pCR}$ and to determine the significance of various factors affecting pCR. Secondary objective was to determine the recurrence rate and patterns of recurrence.

\section{Materials and Methods}

We retrospectively reviewed patients with locally advanced esophageal cancer (middle and lower third) and gastroesophageal junction (Siewert I and II) treated at our hospital from September 2013 to July 2015. These patients were selected

\footnotetext{
How to cite this article: Bajwa HK, Singareddy R, Reddy MM, Raju KA, Rao ST, Rajappa SJ. Preoperative chemoradiation in carcinoma esophagus: Experience from a tertiary cancer center in India. Indian J Med Paediatr Oncol 2018;39:272-5.
}

\author{
Harjot Kaur Bajwa, \\ Rohith Singareddy, \\ Mahendra \\ M Reddy ${ }^{1}$, \\ Krishnam A Raju, \\ Subramanyeshwar \\ T Rao', \\ Senthil J Rajappa ${ }^{3}$
}

Departments of Radiotherapy, ${ }^{2}$ Surgical Oncology and ${ }^{3}$ Medical Oncology,

Basavatarakam Indo American Cancer Hospital and Research Institute, Hyderabad, Telangana, ${ }^{1}$ Department of Preventive and Social Medicine, JIPMER, Puducherry, India

Address for correspondence: Dr. Harjot Kaur Bajwa, Room No 418, $4^{\text {th }}$ Floor, Service Block 5, Basavatarakam Indo American Cancer Hospital and Research Institute, Road No. 10, Banjara Hills, Hyderabad, Telangana, India. E-mail: harjotbajwa1987@ gmail.com

Access this article online Website: www.ijmpo.org DOI: 10.4103/ijmpo.ijmpo_16_16 Quick Response Code:

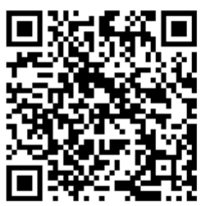


from a larger cohort of operable esophageal cancer patients as they were planned for neoadjuvant chemoradiation followed by surgery. Information on demographic data, initial investigations, and treatment administered were recorded from our hospital database for 50 patients. The pretreatment workup included a physical examination, esophagogastroscopy and biopsy, chest and abdominal computed tomography (CT), and 18F-fluorodeoxyglucose-positron emission tomography, if necessary. After completion of neoadjuvant chemoradiotherapy and before surgery, all patients were imaged with CT abdomen and pelvis. Patients who received preoperative radiotherapy dose of 41.4 Gy with chemotherapy (weekly paclitaxel-carboplatin or cisplatin-5 FU or cisplatin-capecitabine) were included in this study. The outcomes analyzed were $\mathrm{pCR}$, recurrence rate, and recurrence patterns. An ethics committee approval was taken before starting the study. An informed consent was taken from all study participants.

\section{Treatment}

\section{Radiotherapy}

External beam radiotherapy was administered using LINAC $6 \mathrm{MV}$ or $15 \mathrm{MV}$ photons. Radiation technique was based on the discretion of the radiation oncologist. Total dose delivered was 41.4 Gy in 23 fractions of 1.8 Gy, 5 fractions per week. Thirty-nine patients were treated by conventional technique, 4 patients by 3DCRT, and 7 patients by IMRT technique. The gross tumor volume (GTV) was defined by the primary tumor and any enlarged regional lymph nodes. The planning target volume was defined by giving a proximal and distal margin of $4 \mathrm{~cm}$. In case of tumor extension into the stomach, a distal margin of $3 \mathrm{~cm}$ was chosen. A $2 \mathrm{~cm}$ radial margin around the GTV was provided to include the area of subclinical involvement and to compensate for tumor motion and set up variations.

\section{Chemotherapy}

Chemotherapy was administered concurrently on the $1^{\text {st }}$ day of radiotherapy (RT). Only patients who received concurrent chemotherapy regimen of paclitaxel and carboplatin or cisplatin and 5 fluorouracil or cisplatin and capecitabine were included in the analysis.

\section{Surgery}

Surgery was scheduled for 6-8 weeks after preoperative chemoradiotherapy. Complete resection (R0) was characterized by negative margins on microscopic examination. Incomplete resection was determined by the presence of microscopic residual disease (R1) or gross residual disease $(\mathrm{R} 2)$.

\section{Evaluation of response}

Pathologic response evaluation was performed by analyzing surgical specimens. A pCR was defined by the absence of histologically identifiable residual cancer in the tumor and lymph nodes and fibrosis extending through the different layers of the esophagus.

\section{Follow-up}

The patients were followed up postsurgery at 1 month initially, then every 3 months for first 2 years and every 6 months till 5 years and yearly thereafter, to determine recurrence rates, recurrence patterns, and mortality.

\section{Statistical analysis}

Patients were followed up periodically until the last follow-up or death. Postoperative mortality was defined as death on the day of surgery or within 30 days postsurgery. The relationship of various prognostic factors with pCR was computed using Chi-square test and Fischer's exact test SPSS is a statistical software (statistical package for the social sciences). $P<0.05$ was considered statistically significant.

\section{Results}

A total of 50 patients were included in the analysis (females -32 , males -18 ). The median age was 50 years (22-70 years). All patients had ECOG PS $<2$ at the time of starting treatment. The clinico demographic characteristics are summarized in Table 1.

All patients received 41.4 Gy preoperative radiotherapy in 23 fractions. About $60 \%$ of the patients (30/50) received weekly paclitaxel and carboplatin (CROSS protocol), $32 \%(16 / 50)$ patients received cisplatin and 5 fluorouracil,

\begin{tabular}{|c|c|}
\hline Characteristics & Number of patients \\
\hline \multicolumn{2}{|l|}{ Tumor site (\%) } \\
\hline Middle third & $18(36)$ \\
\hline Lower third & $22(44)$ \\
\hline GE junction & $10(20)$ \\
\hline \multicolumn{2}{|l|}{ Histology $(\%)$} \\
\hline Squamous cell carcinoma & $38(76)$ \\
\hline Adenocarcinoma & $12(24)$ \\
\hline \multicolumn{2}{|l|}{ Grade $(\%)$} \\
\hline Well differentiated & $6(12)$ \\
\hline Moderately differentiated & $16(32)$ \\
\hline Poorly differentiated & $28(56)$ \\
\hline \multicolumn{2}{|l|}{ Technique (\%) } \\
\hline Conventional & $39(78)$ \\
\hline 3D CRT & $4(8)$ \\
\hline IMRT & $7(14)$ \\
\hline \multicolumn{2}{|l|}{ Chemotherapy (\%) } \\
\hline Paclitaxel-carboplatin & $30(60)$ \\
\hline Cisplatin-5-fluorouracil & $16(32)$ \\
\hline Cisplatin-capecitabine & $4(8)$ \\
\hline
\end{tabular}


and $8 \%(4 / 50)$ received cisplatin and capecitabine combination chemotherapy. The neoadjuvant chemotherapy details are described in Table 2.

After receiving preoperative chemoradiotherapy, two patients progressed on treatment and did not undergo surgery. These patients were censored at the time of analysis. The remaining 48 patients underwent surgery at a median time of 8 -week postchemo radiation (4-18 weeks). All patients (100\%) achieved R0 resection. pCR (ypT0N0) was achieved in 19 patients (39.6\%). When histology was analyzed separately, $44.7 \%$ of the resected squamous cell carcinoma achieved a pCR whereas only $20 \%$ of adenocarcinomas achieved a pCR. About $48.3 \%$ (14 out of 29) of patients who received preoperative radiotherapy along with weekly carboplatin-paclitaxel achieved a pCR as compared to $25 \%$ pCR rate for cisplatin-5 FU and $33.3 \%$ pCR rate for cisplatin-capecitabine. When the impact of age, gender, histology, tumor site, radiation technique, and chemotherapy was analyzed, none of the factors were significant [Table 3].

At a median follow-up of 12 months, 38 patients were alive, $8 \mathrm{dead}$, and 4 lost to follow-up. All patients who achieved a pCR were alive and disease free at the time of final analysis.

Out of the 8 patients who died, 7 deaths were related to cancer. One patient died due to cerebrovascular accident. The postoperative mortality rate was $0 \%$ as none of the patients died within 30 days of surgery. Isolated locoregional failure was developed in 1 patient. Synchronous local and distant failure occurred in 1 patient. Eight patients ( $80 \%$ of all recurrences) developed distant metastases as the first site of recurrence. Distant sites of metastasis included the lung $(n=4)$, liver $(n=2)$, and cervical lymph nodes $(n=2)$. Overall recurrence rate (ORR) of patients who underwent surgery was $23 \%$.

\section{Discussion}

Preoperative CRT has been used in the treatment of carcinoma esophagus and gastroesophageal junction for decades and has shown good curative effects in local control and prolonged overall survival. In the multi-institutional Phase III study (CROSS trial) ${ }^{[2]}$ which evaluated the benefit of induction therapy using weekly carboplatin and paclitaxel along with 41.4 Gy radiation versus surgery alone, only a quarter of the patients had squamous histology. Median survival was 49 months in the combined therapy arm compared to 24 months in the surgery arm $(P=0.003)$. The overall 5-year survival was much improved in the combined therapy arm $(47 \%$ vs. $34 \%, P=0.003)$. Patients with squamous histology derived a larger benefit.

Achieving a pCR has been known to be a powerful prognostic factor in patients treated with trimodality therapy. Scheer et al. ${ }^{[3]}$ reported that patients with a pCR after neoadjuvant chemoradiotherapy (NCRT) survived at a rate two times higher than that of other patients. Rohatgi et $a{ }^{\left[{ }^{[4]}\right.}$ suggested that failure patterns were correlated with the proportion of residual carcinoma after NCRT, implying worse survival of patients who had more residual disease after NCRT. In the CROSS trial, 29\% patients had a pCR (ypT0N0) after neoadjuvant chemoradiotherapy. One or more pathologically positive lymph nodes were found in $74 \%$ of patients in the surgery arm and in $31 \%$ of those in the CRT plus surgery arm $(P=0.001)$. The pCR rate in our study was $39.6 \%$ in the entire patient cohort, which was higher than that of the patients in the CROSS trial. However, the pCR rate of squamous cell carcinoma patients in our study was $44.7 \%$ whereas only $20 \%$ patients with adenocarcinoma achieved a pCR, which was comparable to the pCR rates achieved in the CROSS trial squamous cell carcinoma and adenocarcinoma patients (49\% and 23\%, respectively). Among the patients who received 41.4 Gy preoperative radiotherapy with weekly paclitaxel and carboplatin (CROSS regimen), the pCR rate was $48.3 \%$. The complete resection rate (R0) in our study was $100 \%$, which was comparable to the $92 \%$ reported by the randomized controlled CROSS trial. Schneider et al. ${ }^{[5]}$ performed a study evaluating the response to trimodality therapy and found that the 3-year survival rate of R0 patients was $54 \%$, while all non-R0 resection patients died within 3 years.

An updated analysis of the CROSS trial group ${ }^{[6]}$ showed a lower local recurrence rate $(34 \%$ vs. $14 \%, P<0.001)$ and lower risk of peritoneal carcinomatosis (14\% vs. $4 \%$, $P<0.001$ ) following neoadjuvant chemoradiation and that squamous cell carcinoma was an independent prognostic variable in the surgery alone group. Most patients diagnosed with locoregional recurrences (LRRs) also developed synchronous distant metastases. Of the patients

Table 2: Details of preoperative chemotherapy regimens

\begin{tabular}{|c|c|c|c|c|c|c|c|}
\hline Chemotherapy & Dose and schedule & 5 cycles & 4 cycles & 3 cycles & 2 cycles & 1 cycle & pCR \\
\hline Paclitaxel & $50 \mathrm{mg} / \mathrm{m}^{2}$ & 26 & 3 & 1 & - & - & $14(48.3 \%)$ \\
\hline Carboplatin & AUC=2 (weekly) & & & & & & \\
\hline Cisplatin & $75 \mathrm{mg} / \mathrm{m}^{2}(\mathrm{D} 1)$ & - & 1 & 6 & 7 & 2 & $4(25 \%)$ \\
\hline 5 fluorouracil & $1000 \mathrm{mg} / \mathrm{m}^{2}(\mathrm{D} 1-\mathrm{D} 4)$ & & & & & & \\
\hline Cisplatin & $40 \mathrm{mg} / \mathrm{m}^{2}(\mathrm{D} 1)$ & 3 & 1 & - & - & - & $1(33.3 \%)$ \\
\hline Capecitabine & 1000 mg (D1-D14) & & & & & & \\
\hline
\end{tabular}

AUC - Area under the curve; pCR - Pathological complete response 


\begin{tabular}{|c|c|c|c|}
\hline Study characteristics & $\begin{array}{c}\text { Total } \\
(n)\end{array}$ & $\begin{array}{c}\text { Pathological complete } \\
\text { response achieved, } n(\%)\end{array}$ & $P$ \\
\hline \multicolumn{4}{|l|}{$\overline{\text { Age (years) }}$} \\
\hline$<50$ & 23 & $11(47.8)$ & 0.263 \\
\hline$\geq 50$ & 25 & $8(32.0)$ & \\
\hline \multicolumn{4}{|l|}{ Gender } \\
\hline Male & 18 & $5(27.8)$ & 0.195 \\
\hline Female & 30 & $14(46.7)$ & \\
\hline \multicolumn{4}{|l|}{ Histopathology } \\
\hline $\begin{array}{l}\text { Squamous cell } \\
\text { carcinoma }\end{array}$ & 38 & $17(44.7)$ & 0.276 \\
\hline Adenocarcinoma & 10 & $2(20 \%)$ & \\
\hline \multicolumn{4}{|l|}{ Tumor site } \\
\hline Middle third & 17 & $9(52.9)$ & 0.311 \\
\hline Lower third & 21 & $6(28.6)$ & \\
\hline GE junction & 10 & $4(40.0)$ & \\
\hline \multicolumn{4}{|l|}{ Radiation therapy } \\
\hline Conventional & 37 & $14(37.8)$ & 0.732 \\
\hline 3D CRT/IMRT & 11 & $5(45.5)$ & \\
\hline \multicolumn{4}{|l|}{ Chemotherapy } \\
\hline Carboplatin-paclitaxel & 29 & $14(48.3)$ & 0.303 \\
\hline CDDP-5-fluorouracil & 16 & $04(25.0)$ & \\
\hline CDDP-capecitabine & 3 & $1(33.3)$ & \\
\hline
\end{tabular}

3D CRT - Three-dimensional conformal radiotherapy; IMRT - Intensity-modulated radiation therapy; GE - Gastroesophageal; CDDP - Cis diamminedichloridoplatinum

undergoing resection, $24 \%$ and $11 \%$ had concurrent LRRs and distant relapses and only $9.3 \%$ and $3.3 \%$ had an isolated LRR in the surgery and CRT plus surgery arms, respectively. Another meta-analysis by Sjoquist et $a .^{[7]}$ on survival after neoadjuvant chemotherapy or chemoradiotherapy for resectable esophageal carcinoma concluded that there was a $13 \%$ absolute difference in survival at 2 years neoadjuvant chemoradiotherapy versus surgery alone, with similar results for different histological tumor subtypes (squamous cell carcinoma vs. adenocarcinoma). An update of this meta-analysis ${ }^{[8]}$ provided strong evidence for a survival benefit of neoadjuvant chemoradiotherapy or chemotherapy over surgery alone in patients with esophageal carcinoma.

In our study, ORR was $23 \%$. Of the ten patients that recurred, 1 (10\%) patient had isolated loco regional failure, 8 patients ( $80 \%$ of all recurrences) developed distant metastases, and $1(10 \%)$ patient had concurrent loco regional and distant failure. At a median follow-up of 9.5 months, 8 patients were dead. Since the median follow-up is short, it was not possible to comment on survival. Future prospective studies with planned preoperative chemoradiation plus surgery involving a more consistent treatment policy across a homogeneous patient cohort and longer follow-up are required.
Our study has certain limitations. First, the concurrent chemotherapy protocols are not uniform in the group of patients treated. Second, the short median duration of follow-up makes any interpretation of recurrences and survival premature. However, this study provides important insights into the pathological responses and recurrence patterns which are indirect predictors of overall survival. We believe that this study is the first study from India reporting outcomes after administration of preoperative chemoradiation as per CROSS trial regimen (41.4 Gy radiotherapy along with weekly paclitaxel and carboplatin).

\section{Conclusion}

In our study, preoperative chemoradiotherapy in carcinoma esophagus was associated with encouraging pCR rates $(39.6 \%)$ and high rates of $\mathrm{R} 0$ resection. $\mathrm{pCR}$ rates were higher among patients receiving CROSS protocol regimen and in squamous cell carcinomas. Most of the recurrences were distant recurrences.

\section{Financial support and sponsorship}

Nil.

\section{Conflicts of interest}

There are no conflicts of interest.

\section{References}

1. Pramesh CS, Karimundackal G, Jivnani S. Squamous cell carcinoma of the oesophagus: The Indian experience. Springer Tokyo 2015. p. 279-303.

2. van Hagen P, Hulshof MC, van Lanschot JJ, Steyerberg EW, van Berge Henegouwen MI, Wijnhoven BP, et al. Preoperative chemoradiotherapy for esophageal or junctional cancer. N Engl J Med 2012;366:2074-84.

3. Scheer RV, Fakiris AJ, Johnstone PA. Quantifying the benefit of a pathologic complete response after neoadjuvant chemoradiotherapy in the treatment of esophageal cancer. Int $\mathrm{J}$ Radiat Oncol Biol Phys 2011;80:996-1001.

4. Rohatgi PR, Swisher SG, Correa AM, Wu TT, Liao Z, Komaki R, et al. Failure patterns correlate with the proportion of residual carcinoma after preoperative chemoradiotherapy for carcinoma of the esophagus. Cancer 2005;104:1349-55.

5. Schneider PM, Baldus SE, Metzger R, Kocher M, Bongartz R, Bollschweiler $\mathrm{E}$, et al. Histomorphologic tumor regression and lymph node metastases determine prognosis following neoadjuvant radiochemotherapy for esophageal cancer: Implications for response classification. Ann Surg 2005;242:684-92.

6. Oppedijk V, van der Gaast A, van Lanschot JJ, van Hagen P, van Os R, van Rij CM, et al. Patterns of recurrence after surgery alone versus preoperative chemoradiotherapy and surgery in the CROSS trials. J Clin Oncol 2014;32:385-91.

7. Gebski V, Burmeister B, Smithers BM, Foo K, Zalcberg J, Simes J, et al. Survival benefits from neoadjuvant chemoradiotherapy or chemotherapy in oesophageal carcinoma: A meta-analysis. Lancet Oncol 2007;8:226-34.

8. Sjoquist KM, Burmeister BH, Smithers BM, Zalcberg JR, Simes RJ, Barbour A, et al. Survival after neoadjuvant chemotherapy or chemoradiotherapy for resectable oesophageal carcinoma: An updated meta-analysis. Lancet Oncol 2011;12:681-92. 